\title{
Numerical Simulations to Assess ART and MART Performance for Ionospheric Tomography of Chapman Profiles
}

\author{
FABRICIO S. PROL ${ }^{1}$, PAULO O. CAMARGO ${ }^{1}$ and MARCIO T.A.H. MUELLA ${ }^{2}$ \\ ${ }^{1}$ Universidade Estadual Paulista/UNESP, Departamento de Cartografia, Rua Roberto \\ Simonsen, 305, 19060-900 Presidente Prudente, SP, Brazil \\ ${ }^{2}$ Universidade do Vale do Paraíba/UNIVAP, Laboratório de Física e Astronomia - IP\&D, \\ Rua Shishima Hifumi, 2911, 12244-000 São José dos Campos, SP, Brazil
}

Manuscript received on February 15, 2017; accepted for publication on April 28, 2017

\begin{abstract}
The incomplete geometrical coverage of the Global Navigation Satellite System (GNSS) makes the ionospheric tomographic system an ill-conditioned problem for ionospheric imaging. In order to detect the principal limitations of the ill-conditioned tomographic solutions, numerical simulations of the ionosphere are under constant investigation. In this paper, we show an investigation of the accuracy of Algebraic Reconstruction Technique (ART) and Multiplicative ART (MART) for performing tomographic reconstruction of Chapman profiles using a simulated optimum scenario of GNSS signals tracked by ground-based receivers. Chapman functions were used to represent the ionospheric morphology and a set of analyses was conducted to assess ART and MART performance for estimating the Total Electron Content (TEC) and parameters that describes the Chapman function. The results showed that MART performed better in the reconstruction of the electron density peak and ART gave a better representation for estimating TEC and the shape of the ionosphere. Since we used an optimum scenario of the GNSS signals, the analyses indicate the intrinsic problems that may occur with ART and MART to recover valuable information for many applications of Telecommunication, Spatial Geodesy and Space Weather.
\end{abstract}

Key words: Grid-based tomography, electron density, ionospheric profile, Chapman function, GNSS, TEC.

\section{INTRODUCTION}

The Global Navigation Satellite System (GNSS) has emerged in recent decades as an effective technology for imaging the morphology and dynamics of the ionosphere. Important contributions of GNSS have been developed by many researches in order to provide valuable information of the

Correspondence to: Fabricio dos Santos Prol

E-mail: fabricioprol@hotmail.com ionosphere in maps of Vertical Total Electron Content (VTEC) (Otsuka et al. 2002, Azpilicueta et al. 2006, Hernández-Pajares et al. 2009, Durmaz and Karslioglu 2015). Despite considerable progress in ionospheric modeling based on GNSS data, a shortcoming of VTEC maps is that the user cannot retrieve the vertical distribution of the electron density in the ionosphere. In those maps, the electron content of the ionosphere is projected on a thin shell located at an altitude of about 350- 
$450 \mathrm{~km}$ above the Earth's surface. Consequently, the accuracy of the ionospheric delay to be used in GNSS positioning is compromised (Brunini et al. 2004) and GNSS data for applications that analyze the vertical morphology of the ionosphere is restricted. However, due to the community's interest in recovering vertical information, tomographic reconstruction techniques linked to GNSS observations began to be used to reconstruct electronic density information.

The geometry of GNSS signals is not completely sufficient to provide a unique and independent solution to the ionosphere by using ionospheric tomographic algorithms (Bust and Mitchell 2008). To overcome this geometric deficiency, constraints or initial conditions of the ionosphere are imposed in the reconstruction method (Wen et al. 2012, Seemala et al. 2014, Prol and Camargo 2016). However, the efficiency of the ionospheric tomography is an issue that depends on the assumptions made in the tomographic reconstruction method. Simulated scenarios have been constructed to retrieve TEC observations with the main goal of assessing the efficiency of the tomographic algorithms using controlled assumptions so that the limitations of the ionospheric tomographic reconstruction methods can be outlined.

Austen et al. (1988) first performed ionospheric tomographic reconstructions using simulated data, showing that the VTEC resulting from the Algebraic Reconstruction Technique (ART) match the VTEC used as reference quite well. Another algebraic algorithm, the Multiplicative Algebraic Reconstruction Technique (MART), was first used by Raymund et al. (1990) for ionospheric imaging. They used a simulated scenario to accurately reconstruct the ionosphere using MART and showed that the reconstruction was highly dependent on the initial guess of the ionosphere. Since then, many other simulations have been conducted to analyze the efficiency of ionospheric tomographic algorithms (Raymund et al. 1994, Howe et al. 1998, Mitchell and Spencer 2003, Thampi et al. 2004, Materassi and Mitchell 2005a, b, Wen et al. 2012, Chartier et al. 2014, Seemala et al. 2014) and for making comparison between ART and MART algorithms for ionospheric studies (Das and Shukla 2011). However, in these simulated studies, the analysis of the efficiency of the algorithms uses climatological models to simulate the ionospheric morphology and/or depends on the real scenario of existing ground-based GNSS receivers used to retrieve the simulated observations of TEC. The climatological models compromise the number of distinct scenarios that can be simulated to represent the ionosphere in regional applications. Also, the GNSS geometry may not be enough for high performance of ART and MART when using real scenarios of ground-based GNSS receivers. Due to the lack of GNSS data for many networks, many voxels of the tomographic system are not illuminated and, then, it may not be possible to obtain a complete understanding of the intrinsic problems of ART and MART for ionospheric tomography.

Two aspects were considered in the analysis of the ART and MART algorithms in this paper: (1) a simulated optimum scenario of GNSS signals was used and; (2) a fully controlled simulation based on the Chapman function described distinct scenarios of the ionosphere. Since we used an optimum scenario for the GNSS signals geometry, the investigation highlights the intrinsic problems that may be expected from the solution obtained from ill-conditioned tomographic systems derived from ground-based GNSS receivers. We then have a clear indication of the efficiency and limitations of ART and MART for the estimation of the Chapman profiles and certain parameters that describe the ionosphere, such as the critical electron density, the peak height, the shape of the ionosphere and the Total Electron Content (TEC). Such parameters have valuable meaning for many applications of 
Telecommunication, Spatial Geodesy and Space Weather, which make the analyses an important specification in the performance of ionospheric tomographic algorithms. Section 2 shows the mathematical formulation of ART and MART. Section 3 presents details of the methodology used to simulate the GNSS signals and the ionosphere. Section 4 presents the results of the numerical simulations and Section 5 shows the conclusions about the use of the algebraic techniques.

\section{ART AND MART FORMULATION}

In Computerized Ionospheric Tomography (CIT), the principal parameter used to describe the ionosphere is the Total Electron Content (TEC), which for GNSS data is expressed as the integral of the electron density $\left(n_{e}\right)$ along the path from the GNSS satellite $(s)$ to the receiving antenna $(r)$, in a column whose cross-sectional area is equivalent to $1 \mathrm{~m}^{2}$. It can be written as (Seeber 2003):

$$
T E C=\int_{r}^{s} n_{e} d s
$$

and for using TEC in CIT, the ionosphere is broken down into a grid of cells (pixels or voxels) and TEC is approximated to a finite sum. Assuming the electron density $\left(n_{e j}\right.$ ) for a cell $j$ and $d_{i j}$ the path length of the GNSS signal $i$ inside the boundaries that intersect the cell $j$, equation (1) can be formulated as (Prol and Camargo 2015):

$$
T E C_{i}=\sum_{j=1}^{J} n_{e j} d_{i j}
$$

where $j$ ranges from 1 to $J$ (number of voxels into the grid) and $d_{i j}=0$ if the signal does not intercept the corresponding cell. The distance $d_{i j}$ may be calculated from the projection of an IPP (Ionospheric Pierce Point) in each point where the signal transverses a grid cell. Therefore, algebraic techniques are used to estimate $n_{e}$ in each cell.
ART works by an iterative process. An initial guess $(\mathrm{K}=0)$ for the electron density $\left(n_{e j}^{0}\right)$ is obtained from initial conditions of the ionosphere, and the electron density for iteration $\mathrm{K}+1$ is then calculated by the following expression (Austen et al. 1988, Pryse et al. 1998):

$$
n_{e j}^{K+1}=n_{e j}^{K}+w \frac{\left(T E C_{i}-\sum_{j=1}^{J} d_{i j} n_{e j}^{K}\right)}{\sum_{j=1}^{J} d_{i j}^{2}} d_{i j}
$$

where $n_{e j}^{K+1}$ is the electron density value obtained from iteration $K+1, w$ is a weighting parameter and $T E C_{i}$ is the TEC observation for signal $i$ usually derived from GNSS data. The $\sum_{j=1}^{j} d_{i} n_{e j}{ }_{e j}^{K}$ term is equivalent to a scan through each cell from the ionospheric grid that calculates the TEC value of the correspondent initial condition of the ionosphere. The initial condition is usually obtained from empirical models of the ionosphere, such as from the International Reference Ionosphere - IRI (Bilitza et al. 2011), to fill in each cell from the CIT grid.

In ionospheric imaging, different versions from ART are commonly applied, such as MART. While ART performs the iterations with a linear formulation, MART is an entropy-optimization algorithm based on the following nonlinear iteration (Raymund et al. 1990, Pryse et al. 1998):

$$
n_{e j}^{K+1}=n_{e j}^{K}\left(\frac{T E C_{i}}{\sum_{j=1}^{J} d_{i j} n_{e j}^{K}}\right)^{w d_{i j} / d_{\max }}
$$

where $d_{\max }$ is the largest path length of the respective signal. Even when $\sum_{j=1}^{J} d_{i j} n_{e j}{ }_{e j}^{K}>T E C_{i}$, MART produces non-negative values so, at a first view, an advantage to using MART, instead of ART, is the guarantee of non-negative values for the reconstructed image.

It is worth mentioning that the weighting parameter is usually determined by empirical experiments and used to control the convergence of the algorithm with $0<w<1$ (Bust and Mitchell 
2008). Pryse et al. (1998) adopted $w=0.2$ with an iterative process that cycles through all ray paths three times for ART and six times for MART. In the present work, the weighting parameter was adopted ten times smaller than used by Pryse et al. (1998), i. e., $w=0.02$, but the iterative process cycles through all ray paths one thousand times for both ART and MART. This strategy was adopted because a smoother solution is expected when reducing the weighting parameter and increasing the number of iterations (Wen et al. 2007). Also, the iterations are performed with a fixed number of times for ART and MART in order to use the same configurations for the comparison between the algorithms.

\section{INVESTIGATIONAL METHOD}

In the present study, we did not seek to analyze any seasonal behavior of the ionosphere, any particular phenomena at a specific region and period or any real scenario of a GNSS network. The intention here is to analyze the differences and limitations of ART and MART for ionospheric imaging of Chapman profiles using a geometry that enables high performance of the algorithms. We therefore created a simulated ionosphere using a Chapman function and a large number of signals with the same geometry as the ground-based GNSS receivers.

A two-dimensional (2D) ionospheric grid was structured with a pixel resolution of $1^{\circ}$ in latitude by $20 \mathrm{~km}$ in altitude and the signals were constructed considering virtual GNSS receivers located at every $5^{\circ}$ in geographic latitude and in the $50^{\circ} \mathrm{W}$ geographic longitude section. The GNSS signals were designed for an elevation angle that ranges from $15^{\circ}$ to $85^{\circ}$ with a $5^{\circ}$ interval. The azimuths were determined equals to $0^{\circ}$ or $180^{\circ}$, making it ideal for two-dimensional case simulations. Figure 1 illustrates the ionospheric grid, the virtual receivers and the coverage area of the signals designed for one receiver.
IRI was used to calculate parameters of a Chapman function. The following formulation was used (Rishbeth and Garriott 1969):

$n_{e}=n_{m} e^{1-z-e^{-z}}$

with:

$z=\frac{\left(h-h_{m}\right)}{H_{s}}$

where the peak height $h_{m}$ and the critical electron density $n_{m}$ were obtained from IRI2012 for each set of pixels that defines an ionospheric profile. In addition, we used a previous least square adjustment procedure to estimate the scale factor $H_{s}$ using observations of electron density $n_{e^{*}}$

Figure 2 shows the input data from IRI (left panel) for day 5, 2014, and the resulting image when performing the Chapman adjustment (right panel). This adjustment approach was performed using observations of electron density derived from IRI and a function model based on equation (5). The design matrix was built by calculating the partial derivative of the electron density in relation to the scale height. The following equation was used:

$\frac{\partial n_{e}}{\partial H_{s}}=n_{m} e^{1-z-e^{-z}}\left(\frac{z}{H_{s}}-z \frac{e^{-z}}{H_{s}}\right)$

where a distinct scale height was iteratively estimated for each set of pixels that describes an ionospheric profile. As a result, electron density retrieved from IRI was not directly used to fill in each pixel of the ionospheric grid. It was then possible to create a fully controlled ionosphere with distinct values of the Chapman parameters $h_{m}$, $n_{m}$ and $H_{s}$ to perform the simulations.

All upcoming analyses were performed using the Chapman image shown in Figure 2 as the initial condition for the ionospheric tomography. Also, we varied the adjusted Chapman parameters to create new images for retrieving TEC observations. These 


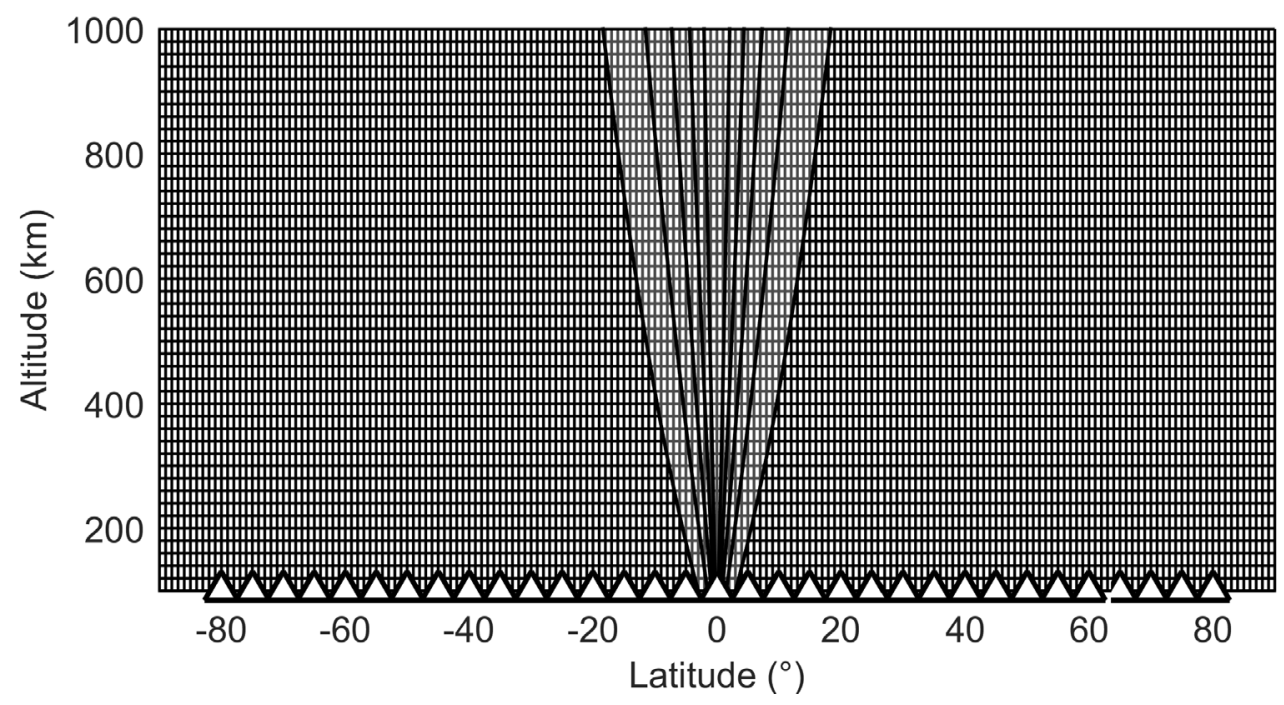

Figure 1 - Ionospheric grid with a spatial resolution of $1^{\circ}$ x $20 \mathrm{~km}$ in latitude by altitude, virtual receivers located at every $5^{\circ}$ (triangles) and the coverage area of the simulated GNSS signals for one receiver.
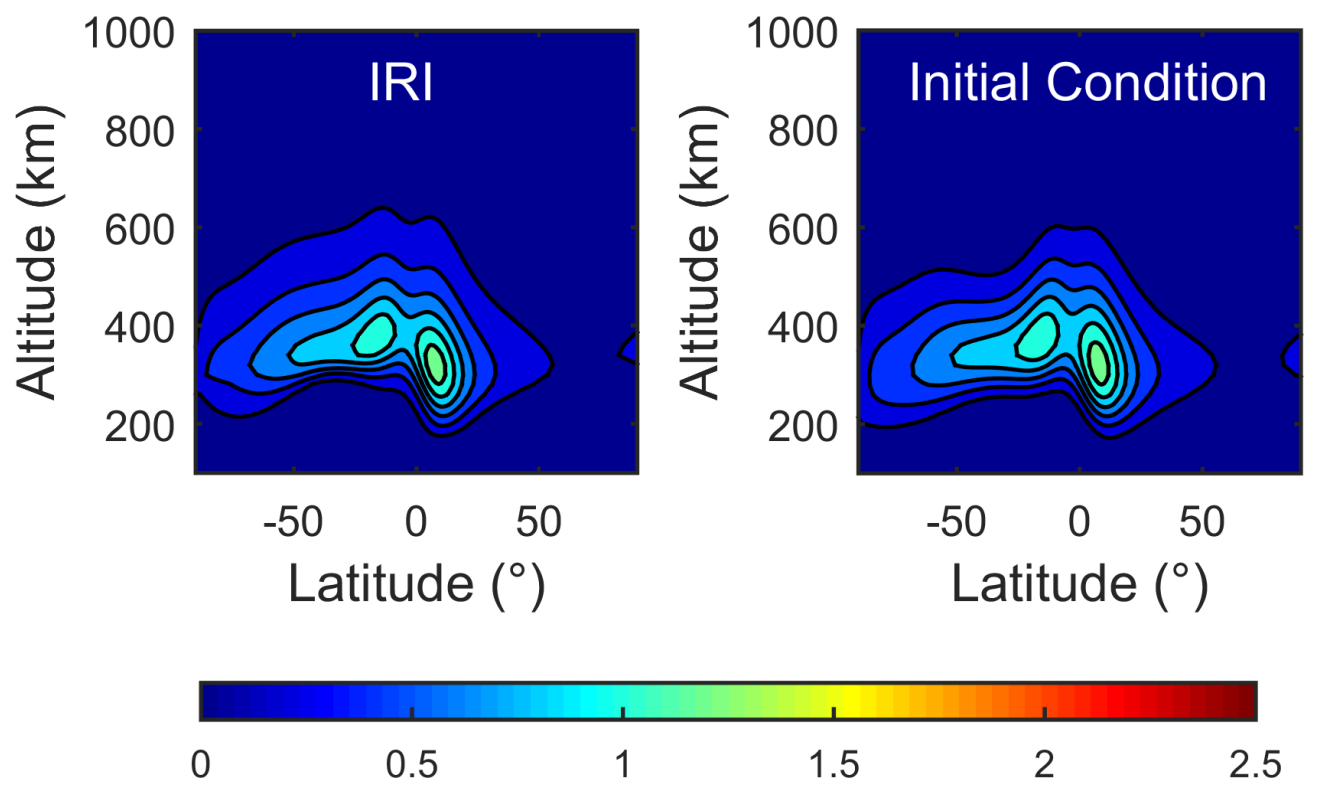

Figure 2 - Electron density obtained from IRI2012 in the left panel and Chapman adjustment to be used as initial condition for ART and MART in the right panel.

new images served as the expected ionosphere to be reconstructed by ART and MART. It was therefore possible to analyze the performance of the algorithms performance using varied values of the Chapman parameters and, consequently, create distinct scenarios.
Visual and numerical analysis was carried out for a total of four configurations, named Cases I, II, III and IV. Cases I and II were developed to analyze the performance of ART and MART for estimating the critical electron density $n_{m}$, Case III analyzed the peak height $h_{m}$ and Case IV was carried out to analyze the scale height $H_{s}$. The visual analyses 
were made to assess the performance of ART and MART for visual interpretations of the ionospheric profiles reconstructed in Cases I, II, III and IV. The numerical analysis was performed to analyze quantitative differences between ART and MART for calculating the parameters $h_{m}, n_{m}$ and $H_{s}$ and also to calculate TEC for each input signal. As mentioned in the previous section, we used the weighting parameter $w$ equals 0.02 and performed the reconstruction using 1000 iterations for all the cases, which allowed us to analyze ART and MART using configurations that enables high performance of the algorithms.

\section{RESULTS OF THE SIMULATIONS}

The next three sections present visual comparisons between ART and MART for the reconstruction of the electron density peak $n_{m}$, peak height $h_{m}$ and scale factor $H_{s}$. The following section then shows the numerical results, presenting an overview of ART and MART performance in estimating $h_{m}, n_{m}$ and $H_{s}$ and also their accuracy in retrieving TEC.

\section{PEAK OF THE ELECTRON DENSITY}

The first analysis (Case I) was constructed using the initial condition shown in Figure 2 (right panel), but the electron density peak $n_{m}$ was doubled for the image to retrieve TEC observations. Figure 3 shows the images used to retrieve TEC and also the resulting reconstruction images from ART and MART algorithms. The limits of the color bar were set equal to the limits used in Figure 2, showing a superior electron density in comparison to the initial condition. We therefore applied the ART and MART algorithms to analyze its performance when the initial condition underestimated of the intensity of the ionosphere.

From Figure 3, we can verify a superior visual performance of MART in comparison with that obtained from ART. It can be seen that, when the observed TEC is larger than the TEC from the initial condition, ART overestimates the electron density at high altitudes and underestimates it at peak height. On the other hand, MART operates with a reasonable performance, showing an electron density peak similar to the observed image.

When the observed value of TEC is lower than the TEC of the initial condition, ART operates in an opposite way to that observed in Case I. This can be seen in the results of Case II (smaller electron density values), where Figure 4 shows that ART underestimates the electron density in the upper altitudes and overestimates in the peak heights. This scenario was produced considering the electron density peak $n_{m}$ of each profile of the image to retrieve TEC equal to half the image of the initial condition. The initial condition of Case II therefore overestimated the intensity of the ionosphere.

In both analyses (Figures 3 and 4), MART reconstructed a more coherent image. However, errors in the reconstruction occurred below $-80^{\circ}$ and above $80^{\circ}$ in geographic latitude because the signals in this region were designed in only one direction, i. e., only signals with the azimuth of $180^{\circ}$ were used below $-80^{\circ}$ and signals with an azimuth equal to $0^{\circ}$ above $80^{\circ}$. Thus, if only one direction occurs with real geometry, the region must be analyzed with special care.

\section{PEAK HEIGHT}

Case III is shown in Figure 5, where the peak height of the image to retrieve TEC was set upwards in comparison with the initial condition. It can be noticed that the image to retrieve TEC observation has more concentration of electron density at high altitudes. This was carried out by multiplying $h_{m}$ by a factor of 1.4 in each profile generating the image for retrieving TEC observations. The 1.4 factor was defined in order to make a significant elevation of the ionosphere in comparison to the initial condition, but without obtaining a non-realistic 


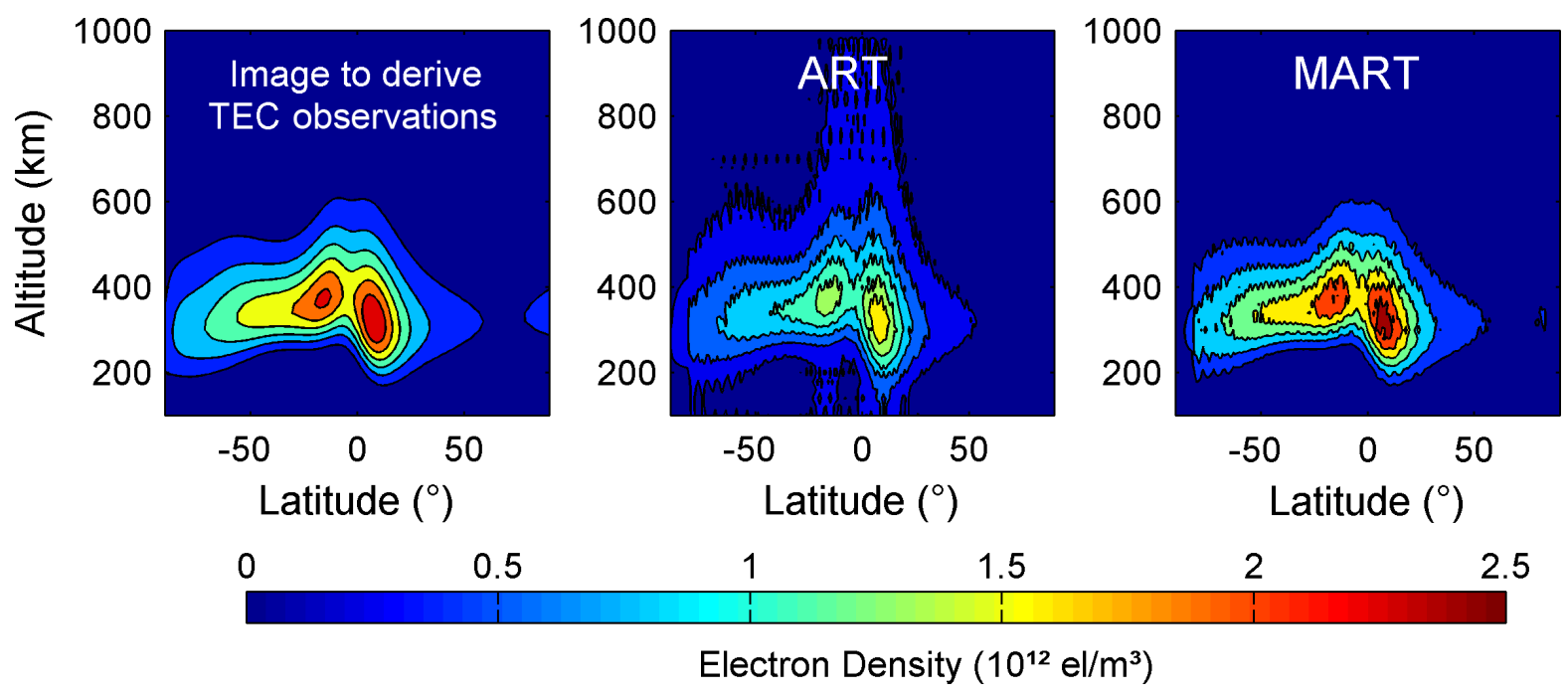

Figure 3 - 2D algebraic technique performance in Case I (larger electron density values).

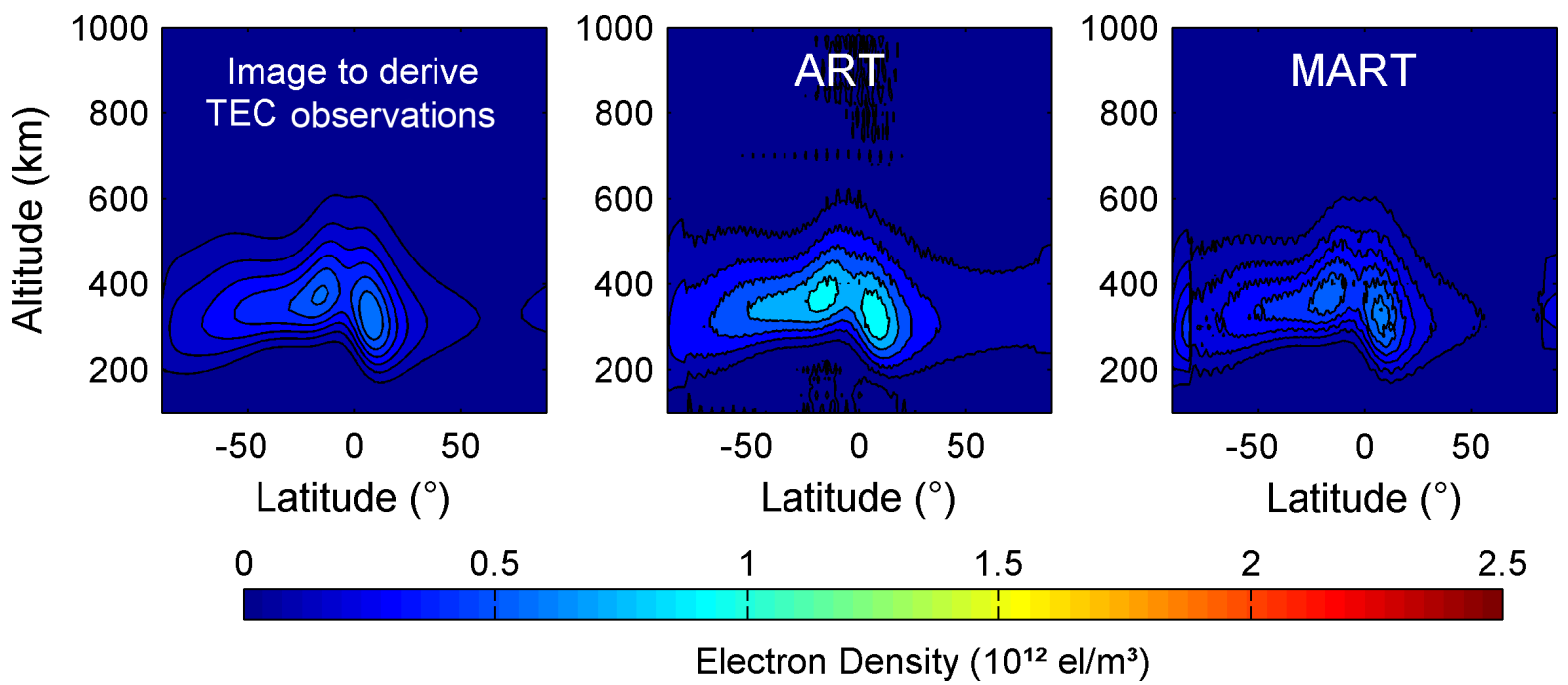

Figure 4 - 2D algebraic technique performance in Case II (smaller electron density values).

peak height for the simulations, such as above 600 $\mathrm{km}$. The maximum peak height obtained for the image to retrieve TEC was about $550 \mathrm{~km}$. In this way, we evaluated ART and MART for mapping the vertical morphology of the ionosphere.

As can be seen, none of the algebraic techniques detected the vertical variation, adding to the limitation in estimating the peak height of the ionosphere. A major difficulty in imaging the uplifted ionosphere arises due to the absence of signals in a horizontal direction and also because the vertical information does not make large changes in the tomographic system, making the observed TEC similar to the TEC from the initial condition. Hence, the vertical information of tomographic reconstruction is inherent to the initial condition used to represent the ionosphere.

Some improvement can be made by including Radio Occultation (RO) data and then increasing the number of signals with different inclinations. 
But special attention is given to ionospheric tomography using GNSS receivers located on the Earth's surface, since it represents the largest number of TEC observations in tomographic reconstructions. It is relevant to say that the GNSS signals must fall within the grid for electron density integration in TEC. However, for regional applications, many RO signals fall outside the grid, which reduces the number of RO signals that can be used in tomographic applications.

\section{SCALE HEIGHT}

Case IV was carried out in order to check the performance of ART and MART to represent the shape of the ionosphere. The image to retrieve TEC observations for Case IV was obtained by doubling the scale factor $H_{s}$ used to build the image of the initial condition of the ionosphere. ART and MART were applied and Figure 6 presents the resulting images.

It can be seen that the shape variation in the image to retrieve TEC observations provided an increase of electron density in the peak height. ART and MART were therefore more sensitive in the regions of the electron density peak. It is also worth mentioning that ART performed better at representing the shape of the ionosphere, what is as expected because MART was developed to scrutinize the entropy of reconstructed images. MART maximizes the intensity of electron density in regions with high entropy and maximum entropy occurs mainly at peak height. Consequently, the electron density peak $n_{m}$ was overestimated in Case IV, mainly in the MART results.

\section{NUMERICAL RESULTS}

An overview of ART and MART performance in estimating $h_{m}, n_{m}$ and $H_{s}$ is presented in Figure 7. The parameters $h_{m}, n_{m}$ and $H_{s}$ were calculated at each latitude based on the ionospheric profiles obtained from the image used for the initial condition (green lines), the image to retrieve TEC observations (black lines), the ART reconstructions (blue lines) and the MART reconstructions (red lines). It is worth mentioning that TEC used as reference in the simulations were obtained with a large range of values, varying from 8 to 172 TEC Units (TECU $-10^{16} \mathrm{el} / \mathrm{m}^{2}$ ) in Case I, 2 to 43 TECU in Case II, 4 to 80 TECU in Case III and 8 to 150 TECU in Case IV.

As can be seen in Figure 7 and similar to the analysis made by Das and Shukla (2011), MART performs better in estimating $n_{m}$. When using $n_{m}$ derived from the image to retrieve TEC observations as reference, we obtained the absolute mean of the discrepancy of MART equal to $0.6710^{11} \mathrm{el} / \mathrm{m}^{3}$ for Case I and $0.2810^{11} \mathrm{el} / \mathrm{m}^{3}$ for Case II. On the other hand, the absolute mean of the discrepancy for ART in Cases I and II was $4.610^{11} \mathrm{el} / \mathrm{m}^{3}$ and $2.410^{11} \mathrm{el} /$ $\mathrm{m}^{3}$, which leads us to the conclusion that MART performed about $87 \%$ better than ART at estimating $n_{m}$. In Case III, both tomographic algorithms presented a similar performance, showing an absolute mean discrepancy of $127 \mathrm{~km}$ and $130 \mathrm{~km}$ for MART and ART, respectively. However, ART presented a better performance in estimating the shape of the ionosphere in Case IV. The absolute mean discrepancy for estimating $H_{s}$ was equal to $85 \mathrm{~km}$ for MART and $55 \mathrm{~km}$ for ART, showing that ART performed about $53 \%$ better than MART.

As an indicator of the quality for estimating TEC using ART and MART, Table I shows the Root Mean Square Error (RMSE) and the absolute maximum discrepancy of TEC. The RMSE of the Reconstruction Techniques (RT) was obtained using the following equation:

$$
R M S E_{R T}=\sqrt{\frac{1}{N} \sum\left(T E C_{o b s}-T E C_{R T}\right)^{2}}
$$

where the TEC reference was defined the same as the TEC observations $\left(T E C_{o b s}\right)$ and $N$ is the number of observations used in each case. 

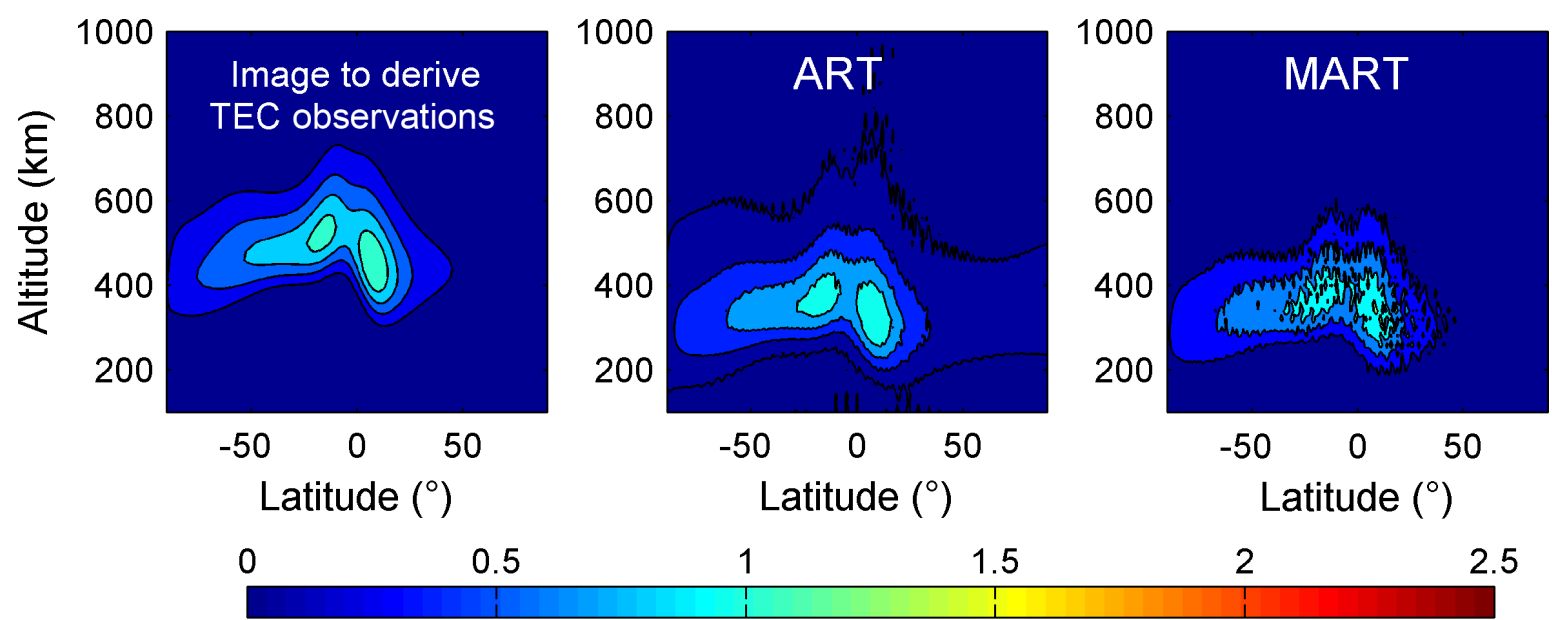

Electron Density $\left(10^{12} \mathrm{el} / \mathrm{m}^{3}\right)$

Figure 5 - 2D algebraic technique performance in Case III (height variation).

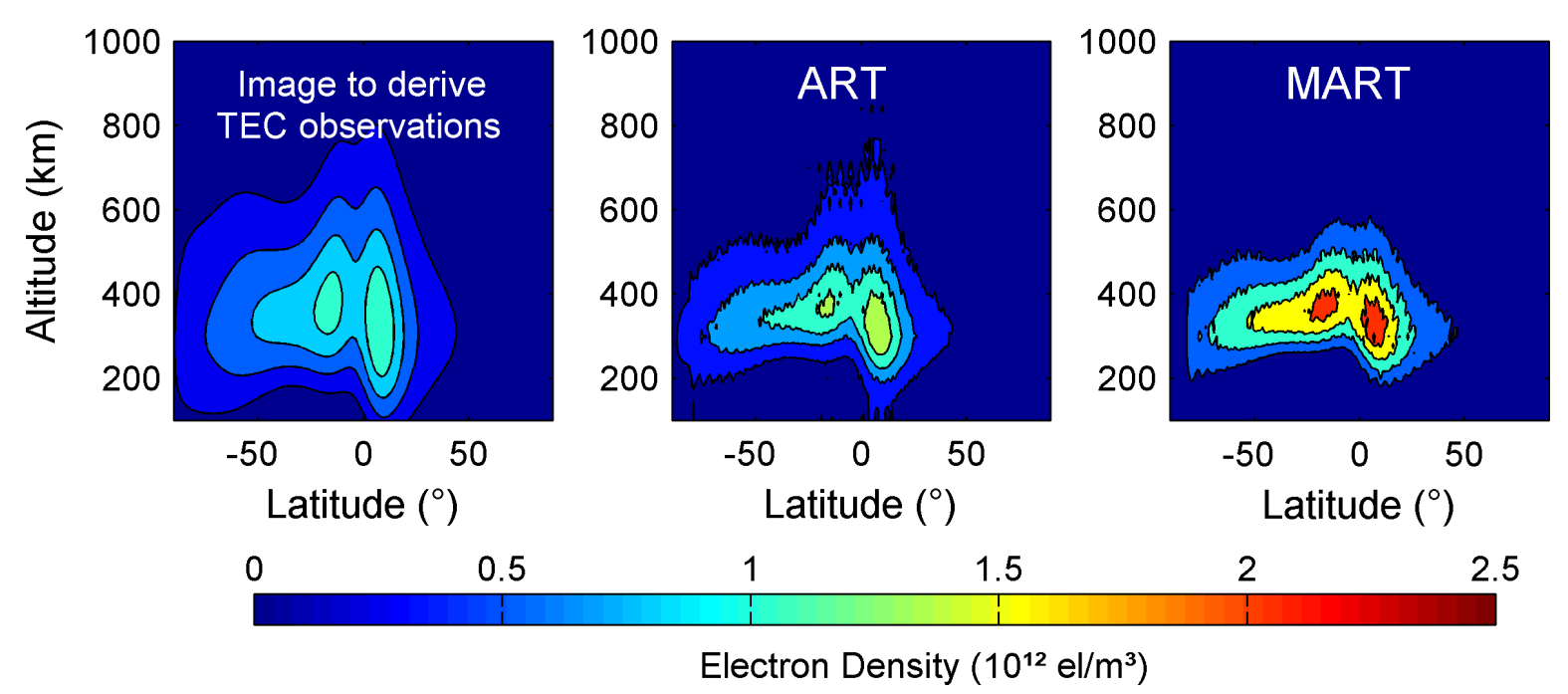

Figure 6 - 2D algebraic technique performance in Case IV (shape variation).

In general, both algorithms were capable of providing images that agree with the input observations within of a few TECU. The greatest discrepancies in the TEC reconstructions using both algorithms were obtained in Case III, which relates to the variations of the peak height, presenting a maximum discrepancy of 0.17 TECU and 0.39 TECU for ART and MART, respectively.

As can be seen in Table I, ART presented lower values in RMSE and in maximum differences. In fact, based on the ART and MART formulations, a better performance is expected for ART in estimating the RMSE, such as discussed by Gordon et al. (1970) when applying the ART and MART algorithms for reconstructing a varied number of distinct objects. The expected ART solution is the one which minimizes the TEC differences between the observed TEC and the TEC obtained through the iterations $\left(T E C_{i}-\sum_{j=1}^{J} d_{i j} n_{e j}\right)$, i.e., ART minimizes the residue that is directly related to the RMSE. On 
the other hand, MART is an entropy-optimization algorithm. The expected solution from MART is the one with the maximum entropy, which occurs when the ratio between the observed TEC and the TEC obtained through the iterations $\left(T E C_{i} / \sum_{i=1}^{J} d_{i j} n_{e j}{ }^{K}\right)$ is close to 1. According to Raymund et al. (1990), by maximizing the entropy, the reconstructed image is less likely to have erroneous artifacts introduced by the reconstruction process. However, the entropy is not directly related to the RMSE. Therefore, despite showing a better performance in visual analysis for Cases I and II, MART showed a worse performance on the TEC estimation.

It is possible to obtain a lower RMSE for MART reconstructions, but more iterations are needed. Figure 8 shows a representative example of the performance of ART and MART to estimate TEC in terms of the iterations, where it is possible to notice that as the number of iterations increases, the TEC RMSE of both algorithms decreases. Also, the ART reconstruction converged with less iterations than the MART reconstruction to obtain a minimum RMSE in the TEC estimation. Therefore, for practical purposes, it is important to consider that ART needs less iterations to obtain a minimum error in TEC estimation when using the same weighting parameter.

\section{CONCLUSIONS}

ART and MART algorithms were analyzed for the ionospheric tomographic reconstruction of Chapman profiles using an optimum scenario of GNSS signals tracked by ground-based receivers and a fully controlled ionospheric simulation. Simulated results were produced to analyze specific cases based on three parameters of the Chapman function. MART presented a better performance in the reconstruction of the electron density peak $n_{m}$ and ART gave a better representation for imaging the shape of the ionosphere described by the scale factor $H_{s}$. Both algebraic techniques reconstructed the horizontal variations well. However, ART and MART presented limitations in representing the vertical morphology of the ionosphere using GNSS geometry with receivers located at the Earth's surface. In general, vertical information described by the peak height $h_{m}$ was highly dependent on the initial condition of the ionosphere. But despite significant errors on estimating $h_{m}$, both algorithms were capable of providing images that agreed well with the input TEC observations, providing a maximum error of 0.4 TECU.

Bearing in mind that we used an optimum scenario of GNSS signals and a fully controlled ionospheric simulation, the limitations pointed out in this paper may serve as an indicator of the intrinsic errors of ART and MART for real scenario of ground-based GNSS receivers. It is reasonable to say that a relevant overview of ART and MART has been provided for future modeling developments. In general, the analyses suggest that the choice of using ART or MART depends on the user application. If the user needs information about peak electron density, MART performed better. However, MART presented a relatively worse performance than ART when estimating the shape of the ionosphere and ART reconstruction converged with less iterations than MART to obtain a minimum error in the TEC estimation. Therefore, the simulated experiments suggest that ART is a more useful algorithm for applications that requires solely TEC information. Also, in

TABLE I

RMSE and maximum discrepancy of TEC estimation by ART and MART. The units are in TECU.

\begin{tabular}{lcccc}
\hline \multirow{2}{*}{ Cases } & \multicolumn{2}{c}{ ART } & \multicolumn{2}{c}{ MART } \\
& RMSE & Max & RMSE & Max \\
\hline Case I & 0.015 & 0.079 & 0.055 & 0.182 \\
Case II & 0.007 & 0.040 & 0.014 & 0.045 \\
Case III & 0.019 & 0.174 & 0.088 & 0.394 \\
Case IV & 0.010 & 0.056 & 0.072 & 0.329 \\
Mean of All & 0.015 & 0.087 & 0.059 & 0.238 \\
\hline
\end{tabular}




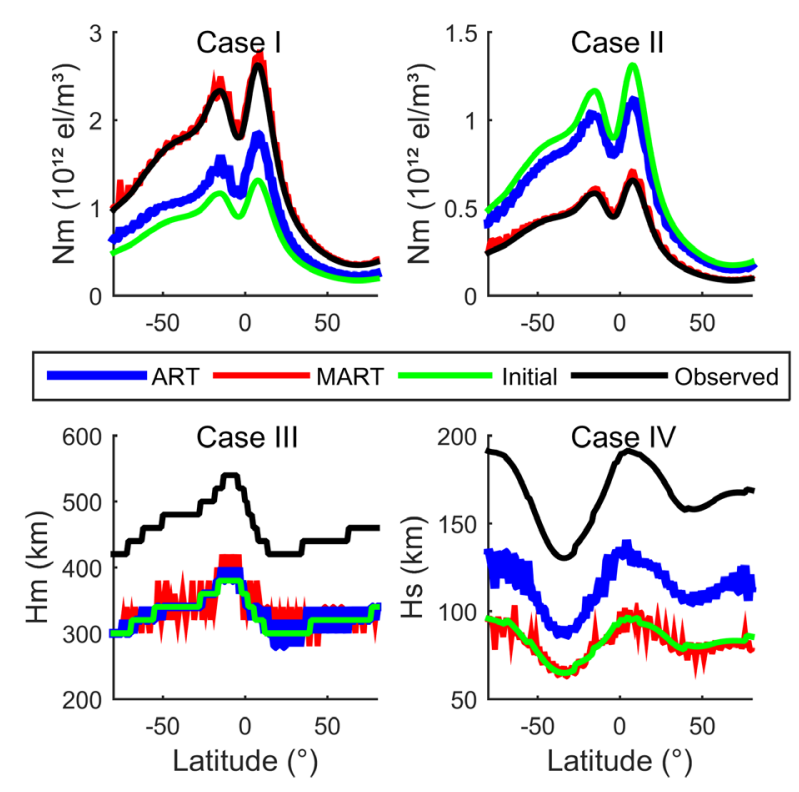

Figure 7 - Performance of ART and MART for estimating $\boldsymbol{n}_{\boldsymbol{m}}$, $\boldsymbol{h}_{\boldsymbol{m}}$ and $\boldsymbol{H}_{\boldsymbol{s}}$ in cases I, II, III, IV.

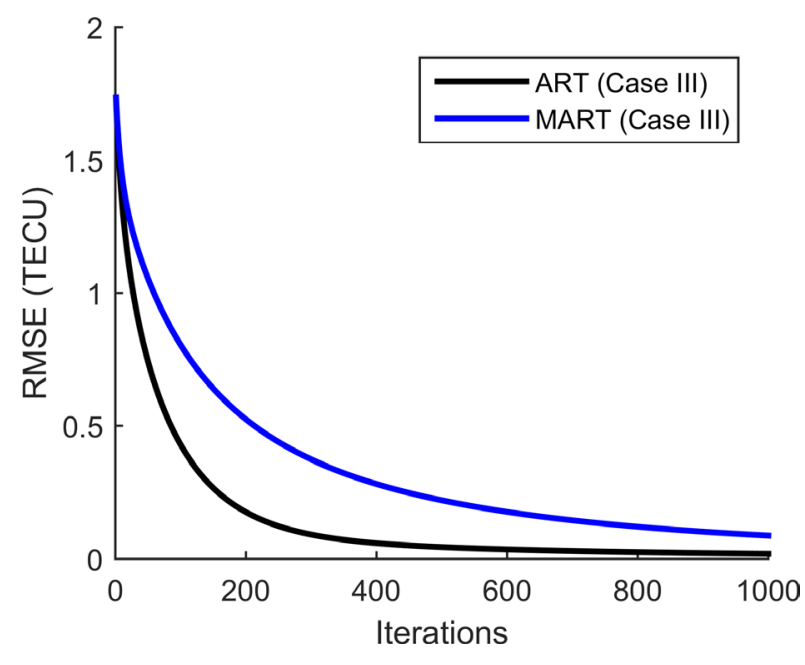

Figure 8 - The RMSE of TEC estimation by ART and MART in each iteration of Case III (height variation), which is the case with maximum RMSE.

future works, RO data may be incorporated into the tomographic reconstructions. A new analysis set may be performed to verify the contribution of RO in ionospheric tomography, mainly for estimating the peak height and scale factor of the ionosphere.

\section{ACKNOWLEDGMENTS}

This work was jointly funded by Fundação de Amparo à Pesquisa do Estado de São Paulo (FAPESP) under grant 2015/15027-7, Coordenação de Aperfeiçoamento de Pessoal de Nível Superior (CAPES) and Conselho Nacional de Desenvolvimento Científico e Tecnológico (CNPq) under grant 2014/09240-7. The authors also acknowledge the use of the IRI model.

\section{REFERENCES}

AUSTEN JR, FRANKE SJ AND LIU CH. 1988. Ionospheric imaging using computerized tomography. Radio Sci 23(3): 299-307.

AZPILICUETA F, BRUNINI C AND RADICELLA SM. 2006. Global ionospheric maps from GPS observations using modip latitude. Adv Space Res 38(11): 2324-2331.

BILITZA D, MCKINNEL LA, REINISCH B AND FULLERROWELL T. 2011. The international reference ionosphere today and in the future. J Geod 85(12): 909-920.

BRUNINI C, MEZA A, AZPILICUETA F, VAN ZELE MA, GENDE M AND DÍAZ A. 2004. A new ionosphere monitoring technology based on GPS. Astrophys Space Sci 290(3): 415-429.

BUST GS AND MITCHELL CN. 2008. History, current state, and future directions of ionospheric imaging. Rev Geophys 46(1): RG1003.

CHARTIER AT ET AL. 2014. Ionospheric imaging in Africa. Radio Sci 49: 19-27.

DAS SK AND SHUKLA AK. 2011. Two-dimensional ionospheric tomography over the low-latitude Indian region: An intercomparison of ART and MART algorithms. Radio Sci 46(2): 1-13.

DURMAZ M AND KARSLIOĞLU MO. 2015. Regional vertical total electron content (VTEC) modeling together with satellite and receiver differential code biases (DCBs) using semi-parametric multivariate adaptive regression B-splines (SP-BMARS). J Geod 89(4): 347-360.

GORDON R, BENDER R AND HERMAN GT. 1970. Algebraic reconstruction techniques (ART) for threedimensional electron microscopy and X-ray photography. J Theor Biol 29(3): 471IN1477-476IN2481.

HERNÁNDEZ-PAJARES M, JUAN JM, SANZ J, ORUS R, GARCIA-RIGO A, FELTENS J, KOMJATHY A, SCHAER SC AND KRANKOWSKI A. 2009. The IGS VTEC maps: a reliable source of ionospheric information since 1998. J Geod 83(3): 263-275. 
HOWE BM, RUNCIMAN K AND SECAN JA. 1998. Tomography of the ionosphere: Four-dimensional simulations. Radio Sci 33(1): 109-128.

MATERASSI M AND MITCHELL CN. 2005a. A simulation study into constructing of the sample space for ionospheric imaging. J Atmos Sol-Terr Phys 67(12): 1085-1091.

MATERASSI M AND MITCHELL CN. 2005b. Imaging of the equatorial ionosphere. Ann Geophys 48: 477-482.

MITCHELL CN AND SPENCER PS. 2003. A threedimensional time-dependent algorithm for ionospheric imaging using GPS. Ann Geophys 46(4): 687-696.

OTSUKA Y, OGAWA T, SAITO A, TSUGAWA T, FUKAO S AND MIYAZAKI S. 2002. A new technique for mapping of total electron content using GPS network in japan. Earth, Planets Space 54(1): 63-70.

PRYSE SE, KERSLEY L, MITCHELL CN, SPENCER PSJ AND WILLIAMS MJ. 1998. A comparison of reconstruction techniques used in ionospheric tomography. Radio Sci 33(6): 1767-1779.

PROLFS AND CAMARGO PO. 2015. Review of tomographic reconstruction methods of the ionosphere using GNSS. Rev Bras Geof 33(3).

PROL FS AND CAMARGO PO. 2016. Ionospheric tomography using GNSS: multiplicative algebraic reconstruction technique applied to the area of Brazil. GPS Solut 20(4): 807-814.

RAYMUND TD, AUSTEN JR, FRANKE SJ, LIU CH, KLOBUCHAR JA AND STALKER J. 1990. Application of computerized tomography to the investigation of ionospheric structures. Radio Sci 25: 771-789.

RAYMUND TD, FRANKE SJ AND YEH KC. 1994. Ionospheric tomography: its limitations and reconstruction methods. J Atmos Sol-Terr Phys 56(5): 637-655.

RISHBETH H AND GARRIOTT OK. 1969. Introduction to Ionospheric Physics. New York: Academic Press, 331 p.

SEEBER G. 2003. Satellite Geodesy: foundations, methods and applications. $2^{\text {nd }}$ ed., Berlin, New York: Walter de Gruyter, 589 p.

SEEMALA GK, YAMAMOTO M, SAITO AAND CHEN CH. 2014. Three-dimensional GPS ionospheric tomography over Japan using constrained least squares. J Geophys Res 119(4): 3044-3052.

THAMPI SV, PANT TK, RAVINDRAN S, DEVASIA CV AND SRIDHARAN R. 2004. Simulation studies on the tomographic reconstruction of the equatorial and lowlatitude ionosphere in the context of the Indian tomography experiment: CRABEX. Annales Geophysicae 22(10): 3445-3460.

WEN D, WANG Y AND NORMAN R. 2012. A new two-step algorithm for ionospheric tomography solution. GPS Solut 16(1): 89-94.

WEN D, YUAN Y, OU J, HUO X AND ZHANG K. 2007. Three-dimensional ionospheric tomography by an improved algebraic reconstruction technique. GPS Solut 11(4): 251-258. 